Presented at $40^{\text {th }}$ Annual Conference of ISCE,

April 15-19, 2015, San Jose, CA

Submitted to Journal of Electrocardiology, May 24, 2015.

Revised MS submitted to Journal of Electrocardiology, August 13, 2015.

\title{
Noninvasive electrocardiographic imaging of chronic myocardial infarct scar ${ }^{\S}$
}

\author{
B. Milan Horáček, PhD, ${ }^{\mathrm{a}, *}$ Linwei Wang, $\mathrm{PhD},{ }^{\mathrm{b}}$ Fady Dawoud, $\mathrm{PhD},{ }^{\mathrm{a}}$ \\ Jingjia $\mathrm{Xu},{ }^{\mathrm{b}}$ John L. Sapp, $\mathrm{MD}^{\mathrm{a}}$ \\ ${ }^{\mathrm{a}}$ Dalhousie University, Halifax, NS, Canada $;{ }^{\mathrm{b}}$ Rochester Institute of Technology, \\ Rochester, NY, USA \\ ${ }^{\S}$ This study was supported by grants from the Canadian Institutes of Health Research, \\ the Heart \& Stroke Foundation of Nova Scotia, the National Institutes of Health \\ NHLBI Award R21HL125998 (to L.W.), and by the National Science Foundation \\ CAREER Award ACI-1350374 (to L.W.).
}

*Corresponding author. c/o Department of Physiology \& Biophysics, 4-P2 Sir Charles Tupper Medical Bldg., 5859 University Avenue, Dalhousie University, Halifax, Nova Scotia, Canada B3H 4H7.

E-mail address: milan.horacek@dal.ca.

Short title: ECG Imaging of Infarct Scar 


\begin{abstract}
Background: Myocardial infarction (MI) scar constitutes a substrate for ventricular tachycardia (VT), and an accurate delineation of infarct scar may help to identify reentrant circuits and thus facilitate catheter ablation. One of the recent advancements in characterization of a VT substrate is its volumetric delineation within the ventricular wall by noninvasive electrocardiographic imaging. This paper compares, in four specific cases, epicardial and volumetric inverse solutions, using magnetic resonance imaging (MRI) with late gadolinium enhancement as a gold standard.

Methods: For patients with chronic MI, who presented at Glasgow Western Infirmary, delayed-enhancement MRI and 120-lead body surface potential mapping (BSPM) data were acquired and 4 selected cases were later made available to a wider community as part of the 2007 PhysioNet/Computers in Cardiology Challenge. These data were used to perform patient-specific inverse solutions for epicardial electrograms and morphology-based criteria were applied to delineate infarct scar on the epicardial surface. Later, the Rochester group analyzed the same data by means of a novel inverse solution for reconstructing intramural transmembrane potentials, to delineate infarct scar in three dimensions. Comparison of the performance of three specific inverse-solution algorithms is presented here, using scores based on the 17segment ventricular division scheme recommended by the American Heart Association.
\end{abstract}

Results: The noninvasive methods delineating infarct scar as three-dimensional (3D) intramural distribution of transmembrane action potentials outperform estimates providing scar delineation on the epicardial surface in all scores used for comparison. In particular, the extent of infarct scar (its percentage mass relative to the total ventricular mass) is rendered more accurately by the 3D estimate. Moreover, the volumetric rendition of scar border provides better clues to potential targets for catheter ablation.

Conclusions: Electrocardiographic inverse solution providing transmural distribution of ventricular action potentials is a promising tool for noninvasively delineating the extent and location of chronic MI scar. Further validation on a larger data set with detailed gold-standard data is needed to confirm observations reported in this study.

Key words: Electrocardiography, myocardial infarction, inverse solution. 


\section{Introduction}

Chronic myocardial infarct scar can be detected, and its location and extent can be determined, by means of a variety of invasive and non-invasive methods, including standard 12-lead electrocardiography $[1,2]$ and cardiac magnetic resonance imaging [3]. Scarring from previous myocardial infarction (MI) constitutes a substrate for ventricular arrhythmias; in patients with postinfarction ventricular tachycardia (VT), accurate delineation of myocardial infarct scar facilitates catheter ablation $[4,5,6,7]$.

For many years electrocardiography has been widely used for detecting and assessing both acute and chronic MI. In particular, modern electrocardiographic imaging technology has emerged as a promising noninvasive approach to mapping the electric activity of the heart and to delineating arrhythmogenic substrates in humans [8]. The present paper reviews recent collaborative studies of the Dalhousie and Rochester groups that are aimed at advancing the ability of noninvasive electrocardiographic imaging to delineate arrhythmogenic substrates. The studies reported here use a common set of electrocardiographic and gold-standard data, providing material for assessing merits and limitations of different algorithms.

\section{Electrocardiographic Assessment of Myocardial Infarction}

It is difficult to obtain detailed information on the extent and location of the damaged myocardium from the standard ECG. Once the repolarization abnormalities (STsegment and T-wave changes) subside after an acute infarction resolves, the $\mathrm{Q}$ waves and a fragmented QRS remain as the only recognized signs of infarction. A fragmented QRS is defined by the presence of an additional $\mathrm{R}$ wave or notching in the $\mathrm{S}$ wave, or the presence of several additional $\mathrm{R}$ waves (fragmentation) in two contiguous leads corresponding to a major coronary artery territory [9].

Various algorithms have been proposed to define location and extent of MI necrosis from the 12-lead ECG. One notable approach to addressing this problem is the Selvester QRS scoring system [1, 10], developed with the aid of a computer model of ventricular activation. The Selvester scoring uses amplitude and duration criteria 
derived from the QRS complexes in 10 of the standard 12 ECG leads (I, II, aVL, aVF, and V1-V6) for a total of 31 points, each equivalent to the necrosis of approximately $3 \%$ of the LV mass. The Selvester scoring system was automated in our Dalhousie lab [11] to facilitate its clinical use. Until recently, myocardial necrosis has been assessed by the Selvester scoring only in the absence of QRS confounders (e.g., bundle branch block, left ventricular hypertrophy, and Wolff-Parkinson-White syndrome). These "no-confounder" criteria have been updated to accommodate cases with hypertrophy and conduction defects $[12,13]$.

\section{MRI Imaging of Myocardial Infarction}

Delayed contrast enhancement magnetic resonance imaging (DCE-MRI) is very effective in determining the presence, location, and extent of MI, in both the acute and chronic stages. This imaging technique works by injecting the subject with an enhancement agent, such as gadolinium, and acquiring a scan within 10 to 20 minutes after injection. Damaged tissue with poor blood perfusion takes a longer time to absorb the contrast agent and is seen as enhanced areas on delayed scanning. An important advantage of the DCE-MRI is that its high spatial resolution allows accurate delineation of 3D transmural infarcted myocardium $[14,15]$. 


\section{Methods}

\section{Patients}

Four patients, who initially presented with acute MI at Glasgow Western Infirmary, without hypertrophy or bundle branch block, were selected for this study from a cohort of 38 patients; all patients provided written informed consent. Each patient underwent clinical assessment, a cardiac MRI for reconstructing ventricular geometry within the chest, a DCE-MRI for 3D delineation of the infarcted region, and 120-lead body-surface potential mapping (BSPM) according to the Dalhousie protocol [16]. Both DCE-MRI and BSPM used in this study were performed at the chronic stage of MI, 6 months after the acute event.

\section{Assessment by Noninvasive Electrocardiographic Imaging}

ECG data were recorded at from 120 locations on the torso sampled at $1 \mathrm{kHz}$ [16], using a Mark 6 acquisition system (BioSemi, Amsterdam, Netherlands). Off-line processing produced an averaged complex for each lead from the 15-second recording. Faulty leads were identified and a 3D interpolation algorithm [17] produced body-surface potentials at 352 nodes of the Dalhousie standard torso. Inverse solutions were obtained by three different methods that calculated epicardial electrograms or volumetric transmembrane potentials (TMP). Individual inverse solutions used patient-specific anatomy derived from MRI data; at the Dalhousie lab, models of the epicardial surface and of the body surface with positions of electrodes were extracted using Amira 4.1 software (Mercury Computer Systems, Chelmsford, MA); at the Rochester lab, volumetric bi-ventricle models of the heart were constructed by custom-written Matlab (Mathworks Inc., Natick, MA) routines. In both labs, all data processing and analysis were performed by custom-written Matlab routines.

The first method of inverse solution, used by the Dalhousie group, calculates epicardial potential distributions from ECG data and patient-specific anatomical data [18]. Briefly, transfer coefficients relating potentials on the epicardial surface to measured body-surface potentials are calculated by using the Radon formula for 
numerical quadrature and then epicardial electrograms are calculated, instant by instant, from body-surface potential distributions by solving the inverse problem using Tikhonov second-order regularization, with the regularization parameter estimated by the $L$-curve method [19].

The second method of the inverse solution - referred to as noninvasive transmural electrophysiological imaging (TEPI) — was introduced by the Rochester group of Wang et al. [20]. This method involves patient-specific reconstruction of TMP dynamics inside 3D ventricular myocardium by means of the Bayesian estimation, where the priors are generated by a Monte-Carlo type simulation of a physiological model of cardiac electrical activity to constrain the TMP solution. The TEPI method is applied to the entire sequence of ECG data, and produces as a solution the spatiotemporal activity of TMP throughout the myocardium. This method was subsequently applied, by the same group, as an imaging modality that provides assessment of MI scar location and extent, as well as detailed delineation of its 3D mass [21]; computational reduction of the method was introduced later [22].

The third method of the inverse solution, which was designed to complement the second one, is Variational Bayesian Electrophysiological Imaging with a totalvariation prior (VB-TV) proposed by Xu et al. [23, 24]. The VB-TV method is well suited to extracting cardiac source activity along the MI-scar border in 3D due to the inherent ability of a total-variation prior to extract strongly localized boundaries between homogeneous regions. It was designed to be applied to selected time instants from the ECG sequence and, for the purposes of infarct delineation, mostly during the ST-segment of the ECG. It produces as a solution the spatial distribution of TMP at specific time instants without temporal dynamics. One of the novel aspects of this method is that the solution uncertainty, assumed to play as important a role as the point estimate, is used to improve the interpretation of solution results.

\section{Scar Localization}

To establish the location and extent of the infarct scar, the three inverse-solution methods described above use different strategies depending on the source model the solution represents.

To interpret inversely-calculated epicardial electrograms generated by the first 
method, waveforms were classified into six morphological categories, depending on the amplitude ratio of the first deflection to the second one. The categories were denoted as Qr, QR, qR, Rs, RS, and rS, where Q/q denotes early downward deflection (Q wave), R/r upward deflection (R wave) and S/s late downward deflection (S wave). Upper and lower case distinguish relative amplitudes of deflections based on a parameter $t(0<t<1)$, which was set at $t=0.25$.

The TEPI method delineates infarct scar in 3D by means of feature extraction from TMP dynamics and by classification of these features. In particular, activation time (AT) and action potential duration (APD) are extracted from each TMP waveform. The former is determined from the maximum first derivative of TMP upstroke, and the latter via repolarization time determined from the maximum second derivative of TMP downstroke. At each ventricular node, scar-related TMP dynamics are specified as changes in AT and APD with respect to their normal values, yielded by model simulation with regular TMP dynamics in healthy hearts, and a clustering technique is used to discriminate between normal and abnormal nodes.

The VB-TV method is applied at only the ST segment of the body-surface ECG data. It first calculates posterior distributions of the TMP, then uses the combination of low mean (low TMP value in necrotic tissue) and low uncertainty (high confidence in the inverse solution) to delineate infarct regions in 3D.

\section{Magnetic Resonance Imaging as Gold Standard}

Magnetic resonance imaging was carried out at Glasgow Western Infirmary with a 1.5-Tesla whole-body scanner (Siemens Sonata, Erlangen, Germany), using a phasedarray chest coil during breath hold and ECG gating. For the accurate assessment of size and location of MI, a DCE-MRI technique was used, as described elsewhere [25]. Briefly, 10 to 20 minutes after intravenous injection of a gadolinium-based contrast agent, sequential short-axis images of the ventricles were acquired, rendering the scarred myocardium as a bright late enhancement area. LV long-axis images were also acquired to provide an additional overview of the MI extent. If regions of late enhancement appeared, they were re-imaged, using 5-mm slice thickness (no gaps). 


\section{Comparison of Electrocardiographic Imaging with DCE-MRI}

Electrocardiographic and MRI data described above were made available to the wide community through PhysioNet/Computers in Cardiology Challenge 2007 [26, 27]. In order to objectively rank entries into the Challenge, George Moody of MIT and Galen Wagner of Duke Medical Center devised a scheme based on the American Heart Association 17-segment diagram [28]; the short-axis images obtained by DCE-MRI were distributed into layers of basal, mid-cavity and apical LV and each image was subdivided into angular AHA segments. Three quantities represented the gold standard: the extent of infarcted myocardium as a percentage of the total mass of the LV, infarct segment numbers as segments with delayed enhancement comprising $>25 \%$ of volume, and the infarct center as the segment containing the approximate center of the region with delayed-enhancement volume $>25 \%$. This reference interpretation of the DCE-MRI images was done independently by two experienced observers and adjudicated by a third. The calculation of infarct extent in studies reported here was refined as follows: the five-point scale was used for every infarcted segment according to the percentage occupied by the infarct mass (1 for 1-25\%; 2 for $26-50 \%$; 3 for $51-75 \%$; 4 for $76-100 \%$ ) and summing the segments weighted by the midpoints $(0.13,0.38,0.63,0.88)$ of this range. Segment overlap (SO) was defined as the percentage of correctly identified segments. 


\section{Results}

Results of electrocardiographic imaging obtained by the three inverse-solution methods are summarized in terms of quantitative metrics in Table 1 and as images in Figs. 1-3. Each method was applied to the same electrocardiographic and anatomical data of four patients (cases 1 to 4 ) whose postinfarction scar was documented by DCE-MRI.

\section{Electrocardiographic Imaging for Case 1}

The infarct scar was located by DCE-MRI to be primarily in the septum (segments 1, $2,3,8,9,13,14$ and 15). The inverse-electrogram morphologies on the epicardial surface in Fig. 1 (first column) show a region of QR pattern (blue) overlaying the anterior interventricular groove and extending towards the left and right ventricles. Figure 2 (top row) shows the true infarct scar (green) located primarily in the septum, particularly in the anteroseptal and inferoseptal segments, extending to the basal and the apical anterior segments and apical inferior segment; the TEPI identified regions (yellow) that overlap (red) anterior and apical segments, but missed the scar's core in two septal segments. Figure 3 (first column) shows true infarct scar (green), and infarct scar as detected by the posterior mean of the VB-TV method (yellow) at the mid-septal region (true positive) with low variance, and extending to false-positive regions with high variance; by accepting only the solution with low variance (with proper thresholding), a clean localization of the 3D scar was obtained, consistent with the yellow contour. Table 1 (Case 1) compares all three methods in terms of quantitative metrics.

\section{Electrocardiographic Imaging for Case 2}

The infarct scar as determined by DCE-MRI extends over inferoseptal and inferior regions (segments $3,4,9,10$ ). Figure 1 (second column) shows the epicardialelectrogram method's estimate of the scar region as corresponding to the areas with a major early downward deflection (largely QR and Qr regions, and to some extent $\mathrm{qR}$ region). The presence of QR-type electrograms over basal right-ventricular regions could be reflecting infarcted myocardium extending over inferoseptal LV (segments 3 and 9). Figure 2 (row $b$ ) shows the true infarct scar (green) and reconstruction by the TEPI method (yellow) that overlaps true-positive segments (red), but over-extends 
into mid anteroseptal and apical segments. Figure 3 (second column) shows the true infarct scar (green), and reconstruction by the VB-TV method (yellow) in basal/mid inferior and basal/mid inferoseptal regions (true positive), consistent with the yellow contour. Table 1 (Case 2) compares all three methods; performance is good for all three.

\section{Electrocardiographic Imaging for Case 3}

Reference DCE-MRI for this patient indicates extensive infarct scar of inferior and inferolateral wall, from basal to apical regions (segments 3-5, 9-12, 15, 16). Scar reconstruction based on epicardial electrograms in Fig. 1 (third column) shows QR morphology overlaying basal as well as middle inferior and middle inferolateral parts of the LV. Figure 2 (row $c$ ) shows the true infarct scar primarily in the inferior and lateral walls (green); reconstruction by the TEPI method (yellow) overlaps truepositive segments (red), but over-extends into anteroseptal segments. Figure 3 (third column) shows the true infarct scar (green), and reconstruction by the VB-TV method (yellow), providing a clean localization in mid-inferior and mid-lateral regions, consistent with the yellow contour. Table 1 (Case 3) compares all three methods; performance is good for all three, with the VB-TV standing out in the overlap measure, achieved by eliminating regions with high variance.

\section{Electrocardiographic Imaging for Case 4}

In this challenging case reference DCE-MRI indicates two areas of infarct: one around the mid-inferior wall (segments 9-11, 15, 17) and the other in the anterobasal region (segment 1). Scar delineation as estimated from epicardial electrograms in Fig. 1 (fourth column) shows a region of QR morphology appearing on basal RV and LV, extending towards the anterolateral LV and a region of qR morphology on the remainder of the inferior surface. Figure 3 (fourth column) shows the true infarct scar (green), and reconstruction by the VB-TV method (yellow), demonstrating its ability to identify both infarcted regions. Table 1 (Case 4) compares all three methods; performances of both volumetric methods are superior to epicardial electrogram classification in their ability to identify two separate scar regions, as well as in estimating the extent of lesions.

In summary, based on qualitative observations involving all four cases, volumetric transmembrane potential imaging methods (M2, M3) are both superior to 
epicardial-electrograms method (M1) in their ability to identify septal scar and the ability to discern non-contiguous scar regions. In quantitative terms, for the extent variable $(n=4)$, Pearson Correlation Coefficients $(\mathrm{CC})$ between three methods (M1, M2, M3) and reference (M0) are $-0.276,0.969,0.985$, respectively, with corresponding $p$ values of $0.7238,0.0312$ and 0.0148 . Comparison of overlap variable $(n=4)$ for methods M1, M2, and M3 shows superior performance of M3, with overlap value of $71.0 \pm 7.9 \%$ (mean \pm SD) compared to $56.9 \pm 10.4 \%$ and 53.1 $\pm 15.0 \%$ for M1 and M2, respectively; paired $t$-test yielded $p$ values of 0.0582 and 0.0510 for M1 vs. M3 and M2 vs. M3 comparisons, respectively. 


\section{Discussion}

Noninvasive electrocardiographic imaging of arrhythmogenic substrates associated with clinical arrhythmias [8] is now accepted as a valuable auxiliary method in cardiac clinical electrophysiology. The aim of the present paper was to show the potential of novel noninvasive transmural electrocardiographic imaging methods to improve pre-ablation planning for patients with scar-related VT.

We have shown that although the delineation of infarct scar from time-varying epicardial potentials can provide some clues about scar-related substrate, the inherent limitation of this approach is its inability to directly image heart-generated electric field within the ventricular wall and in the interventricular septum. Case 1, featuring a septal scar, can serve as an example: although the scar region is manifested on the RV surface as Q waves (Fig. 1, first column), which might imply septal scar, the ambiguity about the true location of the scar cannot be resolved by imaging just the epicardial surface. On the other hand, as apparent from Fig. 3 (first column), the volumetric VB-TV method can isolate the septal scar in Case 1 unambiguously. Case 4, which features two infarct scars, demonstrates the superior ability of volumetric methods, compared to the epicardial electrograms method, to discern these two distinct scar regions (as illustrated in Fig. 3, fourth column, for the VB-TV method). Moreover, it is also apparent from Table 1 that volumetric methods provide better estimates of infarct extent than epicardial electrograms. It should be noted though that the metrics in Table 1 were designed for comparing scar estimates on the epicardial surface and not for volumetric estimates. The infarct extent can be estimated only qualitatively by the epicardial-surface metrics, whereas volumetric methods can measure the infarct extent quantitatively in $3 \mathrm{D}$ as a percentage of infarct nodes in the meshfree spatial lattice comprising the total LV mass [20, 23].

The TEPI and VB-TV methods were developed with complementary advantages: the former describes the temporal dynamics of intramural TMP and is particularly adept in capturing repolarization abnormalities [21], while the latter focuses on the spatial pattern of TMP and takes into consideration uncertainty of the inverse solution [23]. An integration of the two methods can combine their respective advantages, as suggested by a collaborative study of the Dalhousie and Rochester 
groups [29], demonstrating a further improvement in the identification and delineation of scar substrate in comparison with the existing methods. Future work will compare the performance of this new method to those presented in this paper on the same datasets used herein.

\section{Conclusion}

We hypothesized that inversely computed epicardial potentials and/or volumetric intramural transmembrane potentials obtained from noninvasive body-surface potential mapping and anatomical imaging can be used to quantify location and extent of chronic MI scar. The results in terms of three metrics show that volumetric methods are superior to epicardial potential mapping in their ability to delineate infarct scar and to estimate its extent. These improvements of noninvasive electrophysiologic imaging can significantly facilitate preliminary targeting of arrhythmogenic regions before electrophysiologic study and radiofrequency ablation in patients with scar-related VT.

\section{Acknowledgements}

Our special thanks go to George Moody of MIT and to Galen Wagner of Duke University Medical Center, who made 2007 Physionet/Computers in Cardiology Challenge possible. We gratefully acknowledge contributions of Prof. Henry Dargie's Magnetic Resonance Imaging group at Western Infirmary, Glasgow, which provided MRI data for the Challenge and thus made this study possible; individual thanks go to Glasgow group's Tom Martin, Björn Groenning, Heather Murray, Tracey Steedman, John Foster, and Alex Elliot. 


\section{References}

[1] Selvester RH, Wagner GS, Ideker RE. Comprehensive Electrocardiology: Theory and Practice in Health Disease. Ch. Myocardial infarction, pp. 565-629. New York, NY: Pergamon Press, 1989.

[2] Thygesen K, Alpert JS, White HD et al. on behalf of the Joint ESC/ACCF/AHA/WHF Task Force for the Redefinition of Myocardial Infarction, Universal Definition of Myocardial Infarction. J Am Coll Cardiol 2007;50(22):2173-95.

[3] Kim RJ, Albert TSE, Wible JH, Elliott MD, Allen JC, Lee JC, Parker M, Napoli A, Judd RM. Performance of Delayed-Enhancement Magnetic Resonance Imaging With Gadoversetamide Contrast for the Detection and Assessment of Myocardial Infarction: An International, Multicenter, Double-Blinded, Randomized Trial. Circulation 2008;117(5):629-37.

[4] Delacretaz E, Stevenson WG. Catheter ablation of ventricular tachycardia in patients with coronary heart disease. Part I: Mapping. Pacing Clin Electrophysiol 2001;24(8):1261-77.

[5] Delacretaz E, Stevenson WG. Catheter ablation of ventricular tachycardia in patients with coronary heart disease. Part II: Clinical aspects, limitations, and recent developments. Pacing Clin Electrophysiol 2001;24(8):1403-11.

[6] Stevenson WG. Ventricular tachycardia after myocardial infarction: From arrhythmia surgery to catheter ablation. J Cardiovasc Electrophysiol 1995;6:94250.

[7] Ciaccio EJ. Ablation targets in reentrant ventricular tachycardia. Heart Rhythm 2013;10:1117-8.

[8] Rudy Y. Noninvasive electrocardiographic imaging of arrhythmogenic substrates in humans. Circ Res 2013;112:863-74.

[9] Michael MA, Masry HE, Khan BR, Das MK. Electrocardiographic signs of remote myocardial infarction. Progress in Cardiovascular Diseases 2007;50(3):198-208.

[10] Palmeri ST, Harrison DG, Cobb FR, Morris KG, Harrell FE, Ideker RE, Selvester RH, Wagner GS. A QRS scoring system for assessing left ventricular function after myocardial infarction, N Engl J Med 1982;306:4-9.

[11] Horáček BM, Warren JW, Albano A, Palmeri MA, Rembert J, Greenfield JC, Wagner GS. Development of an automated Selvester Scoring System for estimating the size of myocardial infarction from the electrocardiogram. $\mathbf{J}$ Electrocardiol 2006;39:162-8.

[12] Strauss DG, Selvester RH, Lima JAC, Arheden H, Miller JM, Gerstenblith G, Marbán E, Weiss RG, Tomaselli GF, Wagner GS, Wu KC. ECG quantification of myocardial scar in cardiomyopathy patients with or without conduction defects: Correlation with cardiac magnetic resonance and arrhythmogenesis. Circ Arrhythmia Electrophysiol 2008;1:327-36.

[13] Strauss DG, Selvester RH. The QRS complex - a biomarker that "images" the heart: QRS scores to quantify myocardial scar in the presence of normal and abnormal ventricular conduction. J Electrocardiol 2009;42:85-96.

[14] Kim RJ, Fieno DS, Parrish TB, Harris K, Chen E-L, Simonetti OP, Bundy JM, Finn JP, Klocke FJ, Judd RM. Relationship of MRI delayed contrast enhancement to irreversible injury, infarct age, and contractile function. Circulation 1999;100:1992-2002. 
[15] Wu E, Ortiz JT, Tejedor P, Lee DC, Bucciarelli-Ducci C, Kansal P, Carr JC, Holly TA, Lloyd-Jones D, Klocke FJ, Bonow RO. Infarct size by contrast enhanced cardiac magnetic resonance is a stronger predictor of outcomes than left ventricular ejection fraction or end-systolic volume index: prospective cohort study. Heart 2008;94(6):730-6.

[16] Hubley-Kozey CL, Mitchell LB, Gardner MJ, Warren JW, Penney CJ, Smith ER, and Horáček BM. Spatial features in body-surface potential maps can identify patients with a history of sustained ventricular tachycardia. Circulation 1995;92:1825-38.

[17] Oostendorp TF, van Oosterom A, Huiskamp G. Interpolation on a triangulated 3D surface. J Comp Physics 1989;80:331-43.

[18] Horáček BM, Clements JC. The inverse problem of electrocardiography: A solution in terms of single- and double-layer sources on the epicardial surface. Math Biosci 1997;144(2):119-54.

[19] Hansen PC, O'Leary P. The use of the $L$-curve in the regularization of discrete ill-posed problems. SIAM J Sci Comput 1993;14(6):1487-503.

[20] Wang L, Zhang H, Wong K, Liu H, Shi P. Physiological-model-constrained noninvasive reconstruction of volumetric myocardial transmembrane potentials. IEEE Trans Biomed Eng 2010;57(2):296-315.

[21] Wang L, Zhang H, Wong K, Liu H, Shi P. Noninvasive computational imaging of cardiac electrophysiology for 3D infarct, IEEE Trans Biomed Eng 2011;58(4):1033-43.

[22] Wang L. Computational reduction for noninvasive transmural electrocardiographic imaging. Comp Biol Med 2013;43:184-99.

[23] Xu J, Sapp JL, Rahimi Dehaghani A, Gao F, Wang L. Variational Bayesian electrophysiological imaging of myocardial infarction. In: Golland P et al. (Eds.): MICCAI 2014, Part II, LNCS 8674:529-37, 2014.

[24] Xu J, Rahimi Dehaghani A, Gao F, Wang L. Noninvasive transmural electrophysiological imaging based on minimization of total-variation functional. IEEE Trans Med Imag 2014;33(9):1860-74.

[25] Martin TN, Groenning BA, Murray HM, Steedman T, Foster JE, Elliot AT, Dargie HJ, Selvester RH, Pahlm O, Wagner GS. ST-segment deviation analysis of the admission 12-lead electrocardiogram as an aid to early diagnosis of acute myocardial infarction with a cardiac magnetic resonance imaging gold standard. J Am Coll Cardiol 2007;50:1021-8.

[26] PhysioNet/Computers in Cardiology Challenge 2007: Electrocardiographic imaging of myocardial infarction [Online]. Available: http://physionet.org/challenge/2007/.

[27] Dawoud F, Wagner GS, Moody G, Horáček BM. Using inverse electrocardiography to image myocardial infarction - reflection on the PhysioNet/Computers in Cardiology Challenge 2007. J Electrocardiol 2008;41:630-5.

[28] Cerqueira MD, Weissman NJ, Dilsizian V, Jacobs AK, Kaul S, Laskey WK, Pennell DJ, Rumberger JA, Ryan T, Verani MS. Standardized Myocardial Segmentation and Nomenclature for Tomographic Imaging of the Heart: A Statement for Healthcare Professionals From the Cardiac Imaging Committee of the Council on Clinical Cardiology of the American Heart Association. Circulation 2002;105(4)539-42.

[29] Xu J, Sapp JL, Rahimi Dehaghani A, Gao F, Horacek BM, Wang L. Robust transmural electrophysiological imaging: Integrating sparse and dynamic 
physiological models into ECG-based inference. In: Medical Image Computing and Computer-Assisted Intervention (MICCAI), Munich, Germany, October 2015; Lecture Notes in Computer Science, Springer, 2015, accepted. 


\section{Tables}

Table 1: Delineation of infarct scar by three inverse-solution methods compared to delayed-enhancement magnetic resonance imaging (DCE-MRI)

\begin{tabular}{|c|c|c|c|c|}
\hline & $\begin{array}{l}\text { M0: DCE-MRI } \\
\text { Reference }\end{array}$ & $\begin{array}{l}\text { M1: Imaging of } \\
\text { Epicardial } \\
\text { Electrograms } \\
\text { by Dawoud [27] }\end{array}$ & $\begin{array}{l}\text { M2: Volumetric } \\
\text { TMP Imaging } \\
\text { of Wang } \text { et al. }[21] \\
\text { (TEPI) }\end{array}$ & $\begin{array}{l}\text { M3: Volumetric } \\
\text { TMP Imaging } \\
\text { of Xu } \text { et al. } \text { [23] } \\
\text { (TB-TV) }\end{array}$ \\
\hline \multicolumn{5}{|l|}{ Case 1} \\
\hline Centroid & 8 & 9 & 8 & 8 \\
\hline Segments & $1-3,8,9,13-15$ & $2,3,8,9,14$ & $\mathbf{1}, \mathbf{2}, \mathbf{9}, 10, \mathbf{1 3}, \mathbf{1 4}, 16,17$ & $\mathbf{1}-\mathbf{3}, 4,5, \mathbf{8}, \mathbf{9}, \mathbf{1 4}, 17$ \\
\hline Extent & $31 \%$ & $25 \%$ & $24 \%$ & $31 \%$ \\
\hline Overlap & - & $62.5 \%(5 / 8)$ & $62.5 \%(5 / 8)$ & $66.7 \%(6 / 9)$ \\
\hline \multicolumn{5}{|l|}{ Case 2} \\
\hline Centroid & $3 / 4 / 9 / 10$ & 10 & 9 & $3 / 4 / 10$ \\
\hline Segments & $3,4,9,10$ & $\mathbf{3}, \mathbf{4}, 5, \mathbf{9}, \mathbf{1 0}, 11$ & $1, \mathbf{3}, \mathbf{4}, 8, \mathbf{9}, \mathbf{1 0}, 14,16$ & $3,4,10$ \\
\hline Extent & $30 \%$ & $35 \%$ & $32 \%$ & $27 \%$ \\
\hline Overlap & - & $66.7 \%(4 / 6)$ & $50 \%(4 / 8)$ & $75 \%(3 / 4)$ \\
\hline \multicolumn{5}{|l|}{ Case 3} \\
\hline Centroid & $10 / 11$ & 4 & 10 & $10 / 11$ \\
\hline Segments & $3-5,9-12,15,16$ & $3-5,10,11$ & $2, \mathbf{3}-\mathbf{5}, 8, \mathbf{1 1}, \mathbf{1 2}, \mathbf{1 6}, 17$ & $2, \mathbf{3}-\mathbf{5}, 8, \mathbf{9}-\mathbf{1 1}, \mathbf{1 5}, \mathbf{1 6}$ \\
\hline Extent & $52 \%$ & $35 \%$ & $47 \%$ & $40 \%$ \\
\hline Overlap & - & $55.6 \%(5 / 9)$ & $66.7 \%(6 / 9)$ & $80.0 \%(8 / 10)$ \\
\hline \multicolumn{5}{|l|}{ Case 4} \\
\hline Centroid & 15 & 4 & 9 & 15 \\
\hline Segments & $1,9-11,15,17$ & $3-6,9-11$ & $\mathbf{1}, 4,6,7, \mathbf{9}, 14$ & $\mathbf{1}, 6,7, \mathbf{1 0}, \mathbf{1 1}, \mathbf{1 5}, 16, \mathbf{1 7}$ \\
\hline Extent & $14 \%$ & $40 \%$ & $11 \%$ & $20 \%$ \\
\hline Overlap & - & $42.9 \%(3 / 7)$ & $33.3 \%(2 / 6)$ & $62.5 \%(5 / 8)$ \\
\hline
\end{tabular}

Bold face indicates agreement between DCE-MRI reference and the inverse method. Myocardial segments are identified using the 17-segment AHA model [28], with segments coded as follows: 1, basal anterior; 2, basal anteroseptal; 3, basal inferoseptal; 4, basal inferior; 5, basal inferolateral; 6 , basal anterolateral; 7 , mid anterior; 8 , mid anteroseptal; 9, mid inferoseptal; 10, mid inferior; 11, mid inferolateral; 12, mid anterolateral; 13, apical anterior; 14, apical septal; 15, apical inferior; 16, apical lateral; 17, apex. Extent, the percentage of infarct in the ventricular mass; Overlap, segment overlap (SO) measured as the percentage of correctly identified infarct segments. 


\section{Legends}

Figure 1: Delineation of infarct scar for cases 1 to 4 by means of epicardialelectrogram-morphology method of Dawoud [27]. Anterior and inferior views of the epicardial surface, with regions featuring electrograms of six types: Qr (yellow), QR (blue), qR (red), Rs (black), RS (green), and rS (cyan). In case 1, scar was located primarily in the septum and manifested as dominant Q-waves over the right ventricle (yellow) and anterior interventricular groove (blue), covering anteroseptal $(2,8)$ and inferoseptal $(3,9)$ segments. In case 2 , scar located in the inferior and inferoseptal regions was manifested as $\mathrm{Q}$-waves over the inferobasal region $(\mathrm{QR}$, blue) and mid inferior region (qR, red), covering inferior $(4,10)$ and inferoseptal $(3,9)$ segments. In case 3, scar located in the inferior and lateral wall was manifested as Q-waves over the basal and mid inferolateral regions ( $\mathrm{QR}$, blue; $\mathrm{qR}$, red), covering segments 3, 4, 5, 10 , and 11 . In case 4, there were two separate scars; smaller one located in the anterobasal region (segment 1) and the larger located in the mid-inferior and apical regions. The latter scar was manifested on the inferior wall as Q-waves over the inferobasal (QR, blue) and mid inferior regions (qR, red), covering basal (3-6) and mid-inferior (9-11) segment.

Figure 2. Delineation of infarct scar for cases 1 to 3 yielded by the TEPI method of Wang and co-workers [22]. Scar identified by the accelerated TEPI method is depicted in yellow, and the true scar is in green; overlaps are in red. The left and right columns show, respectively, meshfree and oblique volumetric representation of the ventricles. Case 1 (row $a$ ) had scar located primarily in the septum, particularly in the anteroseptal and inferoseptal segments, extending to the basal and apical anterior segments and the apical inferior segment; overlap covers anterior and apical segments, but misses the core in two septal segments. Case 2 (row $b$ ) had scar located primarily in the inferior and inferoseptal segments; overlap covers these regions, but over-extends to the mid anteroseptal and apical segments. Case 3 (row $c$ ) had scar primarily in the inferior and lateral wall; overlap covers these regions, but overextends into anteroseptal segments. 
Figure 3. Delineation of infarct scar for cases 1 to 4 yielded by the VB-TV method of $\mathrm{Xu}$ and co-workers [23]. Rows, from top to bottom, show: estimated mean action potential maps, variation maps, and infarct maps based on the combined mean and variation values (with yellow contours delineating the core of the infarct as determined from DCE-MRI). Case 1 had scar (green) in the septum; the posterior mean detected it at the mid-septal region (true positive) with low variance, and extended to false-positive regions with high variance; by combining both the posterior mean and variance of the solution, a clean delineation of the 3D scar was obtained, consistent with the yellow contour. Case 2 had scar in the inferior and inferoseptal segments (note that the heart is rotated to show the inferior wall); elimination of regions with high variance reduced false-positive regions, yielding a clean delineation in basal/mid inferior and basal/mid inferoseptal regions, consistent with the yellow contour. Case 3 had scar in the inferior and lateral wall; elimination of regions with high variance reduced false positives, yielding a clean delineation in the mid-inferior and mid-lateral regions, consistent with the yellow contour. Case 4 featured two separate scars: the smaller one in the anterobasal region and the larger in the midinferior and apical regions. The VB-TV method demonstrated the unique ability to identify both scars. 
Figures

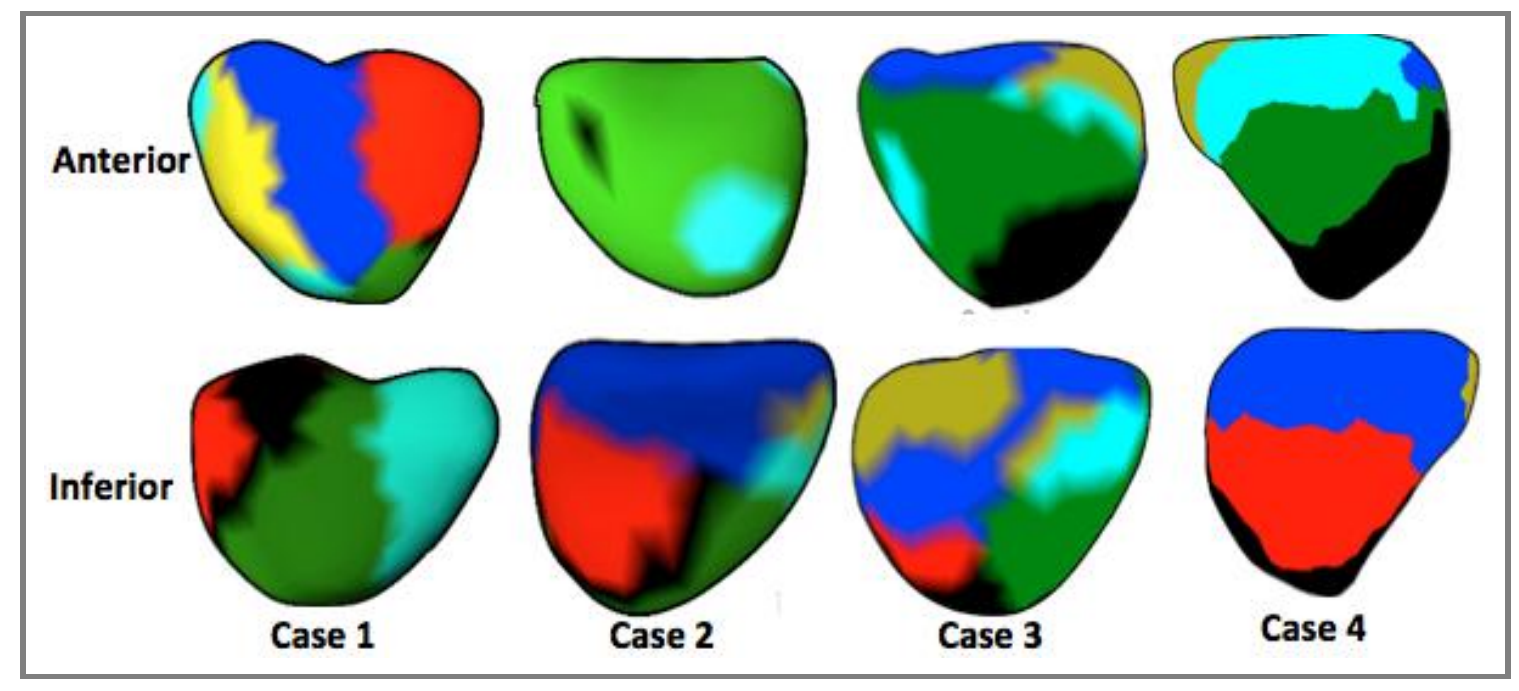

Figure 1 


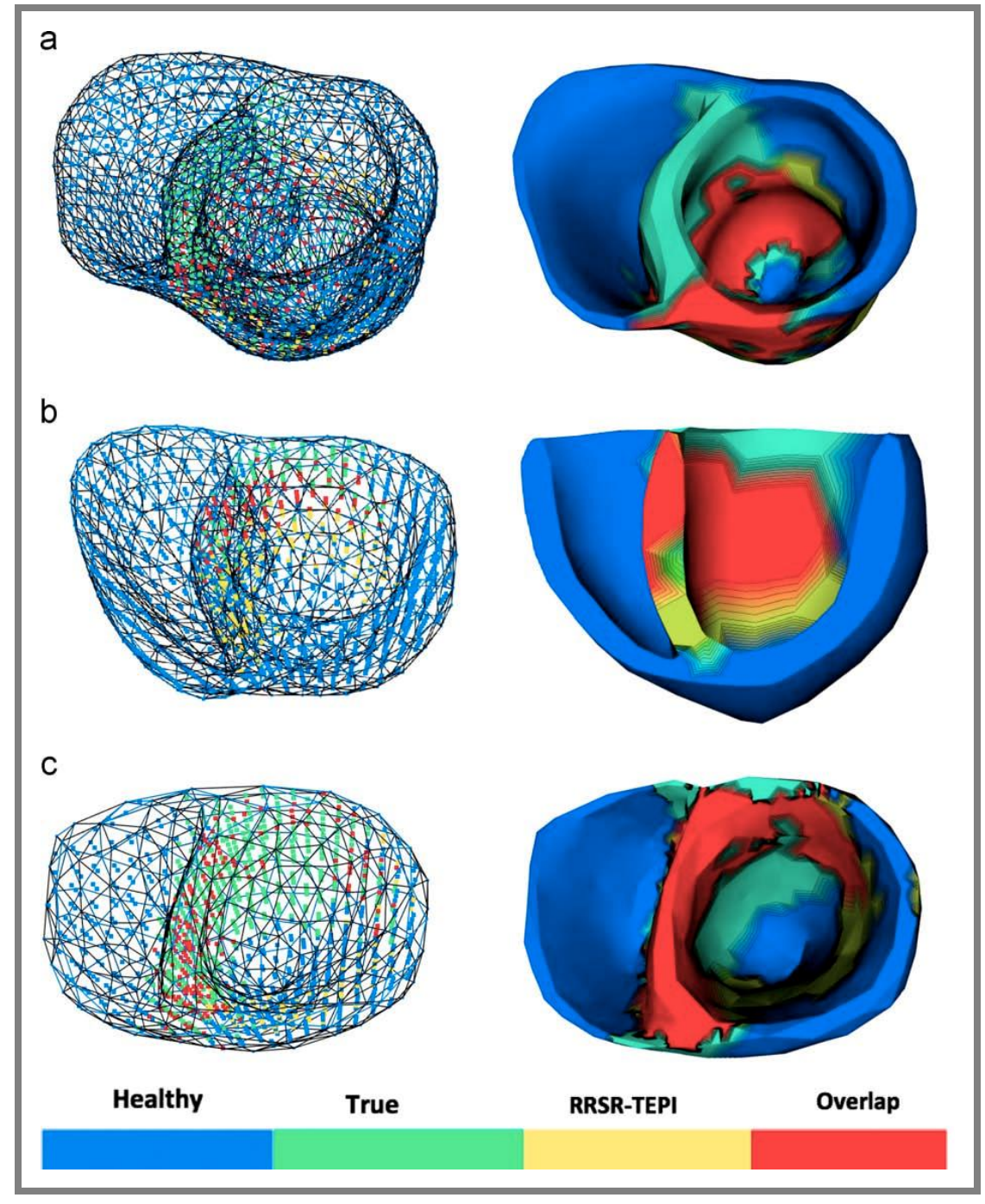

Figure 2 


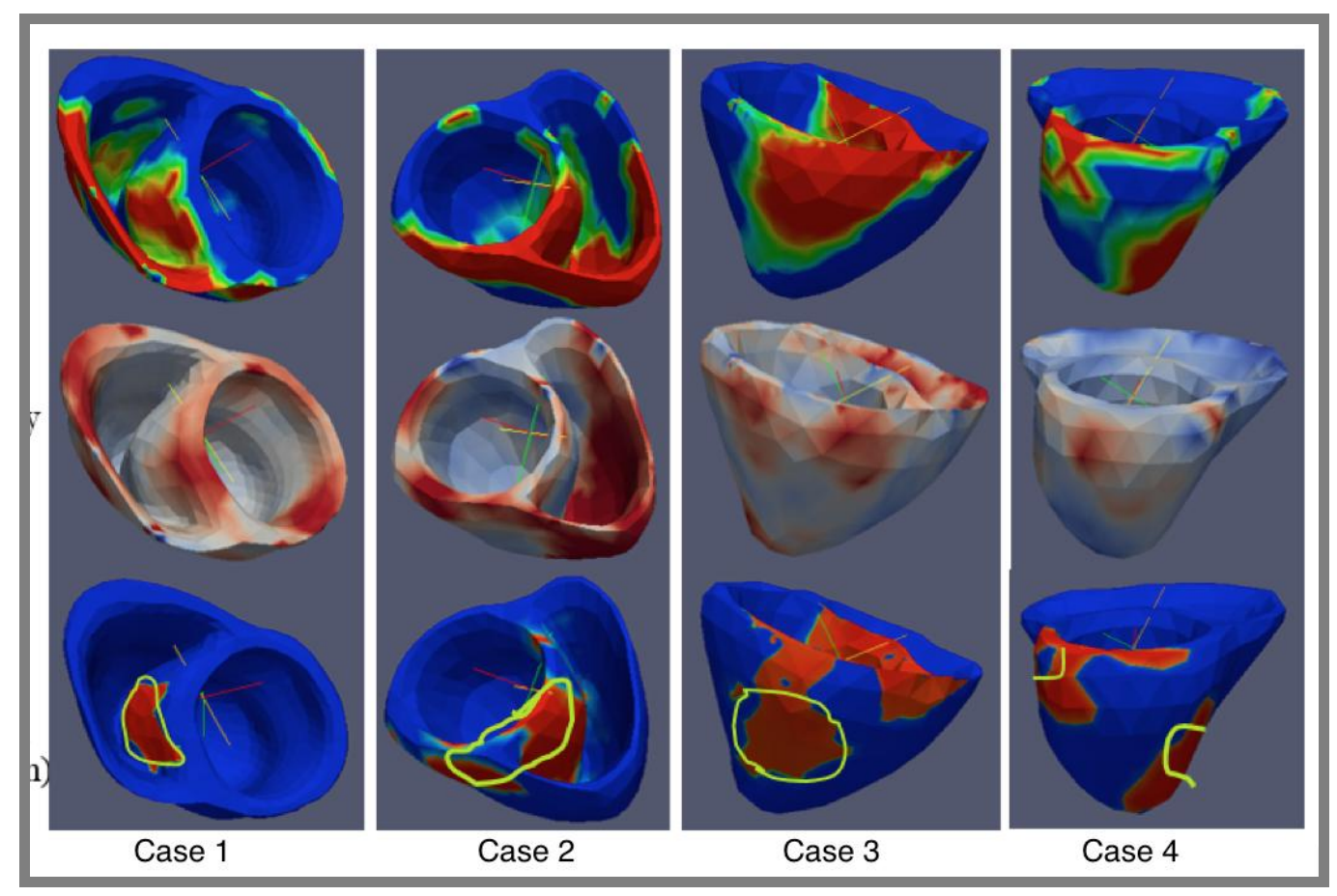

Figure 3 